Original Article

\title{
Intramedullary nailing for treatment of forearm non-union: Is it useful? - A case series
}

\author{
Rocco De Vitis ${ }^{\mathrm{a}, \mathrm{b}, \mathrm{c}}$, Marco Passiatore ${ }^{\mathrm{a}, \mathrm{c}, *}$, Vitale Cilli $^{\mathrm{d}}$, Jacopo Maffeis ${ }^{\mathrm{e}}$, Giuseppe Milano ${ }^{\mathrm{e}}$, \\ Giuseppe Taccardo ${ }^{\mathrm{a}, \mathrm{b}, \mathrm{c}}$
}

${ }^{a}$ Fondazione Policlinico Universitario A. Gemelli IRCCS, Roma, Italy

${ }^{\mathrm{b}}$ Università Cattolica del Sacro Cuore, Roma, Italy

${ }^{\mathrm{c}}$ Istituto di Clinica Ortopedica, Italy

d Centre Hôpitalier de la Haute Senne, Soignies, Belgium

${ }^{\mathrm{e}}$ Università degli studi di Brescia, Italy

A R T I C L E I N F O

\section{Keywords:}

Forearm non-union

Non-union

Intramedullary nailing

Forearm pseudarthrosis

Bone graft

Ulna

Radius

\begin{abstract}
A B S T R A C T
Introduction: Intramedullary osteosynthesis is often considered a second-order method for treatment of forearm non-union.

Methods: We evaluated clinical and functional results from 49 patients affected by radio and/or ulna non-union treated using intramedullary nailing, with possible tricortical autologous bone grafting. Healing rate (primary outcome), healing time and functional status have been assessed.

Results: Healing was observed in 46 cases (93.9\%), the average healing time was 6.3 (SD \pm 2.5 ) months. Excellent result (Anderson score) was reached in 38 cases (77.6\%), satisfactory in 7 cases (14.3\%).

Conclusions: Forearm non-unions represent a therapeutic challenge. Intramedullary nailing is a successful option of treatment.
\end{abstract}

\section{Introduction}

Prevalence of forearm non-union as a complication of diaphyseal fractures is reported varying from $2 \%$ to $10 \% \cdot{ }^{1-4}$ Surgical treatment of forearm non-union should provide bone stability, and restoration of proper length and relationships between radius and ulna, to achieve complete bone healing. Moreover, range of motion (ROM) in both flexion-extension of the elbow and wrist, and pronation-supination should be restored to optimize functional recover. ${ }^{5}$

Several studies reported on a wide variety of surgical options for the treatment of forearm non-unions. ${ }^{6-18}$ Compression plate fixation and autologous bone grafting is the most popularized surgical procedure. However, the success rate of this procedure is variable from 90 to $100 \% .^{3,6,7,11,15,16}$ and there is still debate regarding the efficacy and safety of the procedure. Intramedullary nailing of the forearm has been suggested as a valid option for the treatment of forearm non-union. Potential advantages advocated for this technique are: mini-invasiveness with a very small incision and, if is possible, missed exposure of non-union and integrity of soft tissues and extremely contained cost in comparison to the others technique. However, few studies focused on the effectiveness and adequacy of intramedullary nailing. ${ }^{8,9,12-14}$ The purpose of the present study was to assess the outcome of intramedullary nailing in a cohort of patients affected by forearm nonunion. The hypothesis of the study was that the outcome of intramedullary nailing procedure for the treatment of forearm non-union is comparable to that reported in the literature with the use of a reference standard, such as open reduction and internal fixation (ORIF) using a compression plate fixation and autologous bone graft. ${ }^{3,6,7,19,20}$

\section{Materials and methods}

The present study was designed as a retrospective cohort study and took place between March 1993 and January 2012. The study was in accordance with the principles of the Declaration of Helsinki. Written informed consents were obtained in all patient before the surgery.

\subsection{Participants}

A total of 58 patients were consecutively evaluated. For this study, we enlisted 49 patients who accepted our invitation to enter the study and who signed an agreement disclosure form. All men and women affected by meta-diaphyseal fracture non-union of the ulna, radius or

\footnotetext{
*Corresponding author. Istituto di Clinica Ortopedica., Fondazione Policlinico Universitario A. Gemelli IRCCS, Via Giuseppe Moscati 31, 00168, Roma, Italy.

E-mail address: passiatore.m@gmail.com (M. Passiatore).
} 
both bones were eligible for inclusion. Diagnosis of fracture non-union was established when no radiographic signs of healing observed at a minimum of 6 months after the initial treatment. ${ }^{21}$ Exclusion criteria were: occurrence of other fractures in the same limb at the time of the primary injury, inability or unwillingness to sign informed consent, and Workers' Compensation claims.

\subsection{Associate injuries}

The following associate pathologic conditions were observed: breakage of the previously implanted hardware in 4 cases (8.2\%), palsy of the radial nerve in 4 cases (8.2\%); neuroma of the radial nerve in one case $(2 \%)$; palsy of the median nerve as outcome of a Volkmann's syndrome in one case (2\%); local osteomyelitis in 3 cases $(6.1 \%)$; concomitant misdiagnosed chronic inveterate tran-scaphoid perilunate dislocation in one case (2\%), and ulno-carpal conflict for shortening of the radius in 4 cases $(8.2 \%)$.

\subsection{Interventions}

All the patients underwent internal fixation of fracture non-union with stainless steel nails (Rush's nail) from 3.5 to $5 \mathrm{~mm}$ in diameter and varying in length. The Rush's nail is relatively flexible and has a curved extremity with a "curl", which confers rotational stability to the implant.

For radius fixation, the nail was plunged in a retrograde way. A $20 \mathrm{~mm}$ longitudinal incision was performed on the dorsal aspect of the wrist, laterally to the Lister's tubercle, passing between the extensor carpi radialis brevis (ECRB) and extensor carpi radialis longus's (ECRL) tendons, having spread along the extensor of the thumb (EPL). The nail was initially introduced with an angle of approximately $45^{\circ}$ respect to the dorsal cortex on the sagittal plane, and then it was oriented into the medullary canal and plunged along the longitudinal axis of the radius (Fig. 1).

For ulna fixation, the nail was plunged in an antegrade way. A $10 \mathrm{~mm}$ longitudinal midline incision was performed over the central part of the olecranon. The nail was introduced by a trans-tricipital approach and parallel to the longitudinal axis of the ulna (Figs. 2 and 3).

When medullary canal was obliterated without clear radiographic signs of atrophy or bone loss at the non-union site, closed fixation was preceded by intramedullary drilling with a Steinmann's sharp-tip pin. Open procedure was performed when necessary. Particularly, previously implanted hardware was removed; non-unions showing radiographic signs of atrophy were treated by open debridement; bone loss with shortening of one or both forearm's bones was treated by tricortical bone grafting harvested from the contralateral iliac crest to reestablish proper bone length. Closed reduction and internal fixation were achieved in all the other cases. After surgery, all the patients were immobilized in a closed brachio-antebrachio-metacarpal (BAM) cast with elbow flexed at $90^{\circ}$ and neutral pronation-supinationation for 30 days. After cast removal, the patients were evaluated postoperatively every month by clinical and radiographic examination to assess callus formation and non-union healing.

Concomitant or subsequent treatment of coexisting pathological conditions was performed when necessary. In the only case in which there was a concomitant palsy of the median nerve and the results of a Volkmann's syndrome was performed a neurolysis of the median nerve and wrist arthrolysis. In the only case where there was a concomitant misunderstood inveterate trans-scaphoid perilunate dislocation was performed a proximal carpal row resection.

In three cases of palsy of the radial nerve neurolysis produces a relative comfort. In a case of radial nerve palsy were necessary muscletendinous transfer: pronator rotondus pro extensor carpis, ulnar flexor carpis ulnaris pro extensor comunis digitorum, palmar gracilis pro extensor pollicis longus. We wait 12-18 months before intervening in cases previously complicated by osteomyelitis. In this period patient was subjected to antibiotic therapy and radiographic controls. Additional treatment with shockwave therapy was performed when delayed healing was observed.

\subsection{Outcome measurements}

The primary outcome of the study was assessment of non-union healing rate. Secondary outcomes were timing from surgery to healing, evaluation of global anatomical and functional status of the operated limb through Anderson score and DASH and Work-DASH score. Nonunion healing was assessed on repeated standard radiographic views according to criteria for evaluation of callus formation established by Anderson et al. ${ }^{5,6}$

According to Anderson and colleagues an excellent result consists in healed fracture and $<10^{\circ}$ reduction of wrist and elbow ROM and $<$ $25 \%$ reduction of forearm pronation-supination ROM, a satisfactory result consists in healed fracture and $<20^{\circ}$ reduction of wrist and elbow ROM and $<50 \%$ reduction of forearm pronation-supination

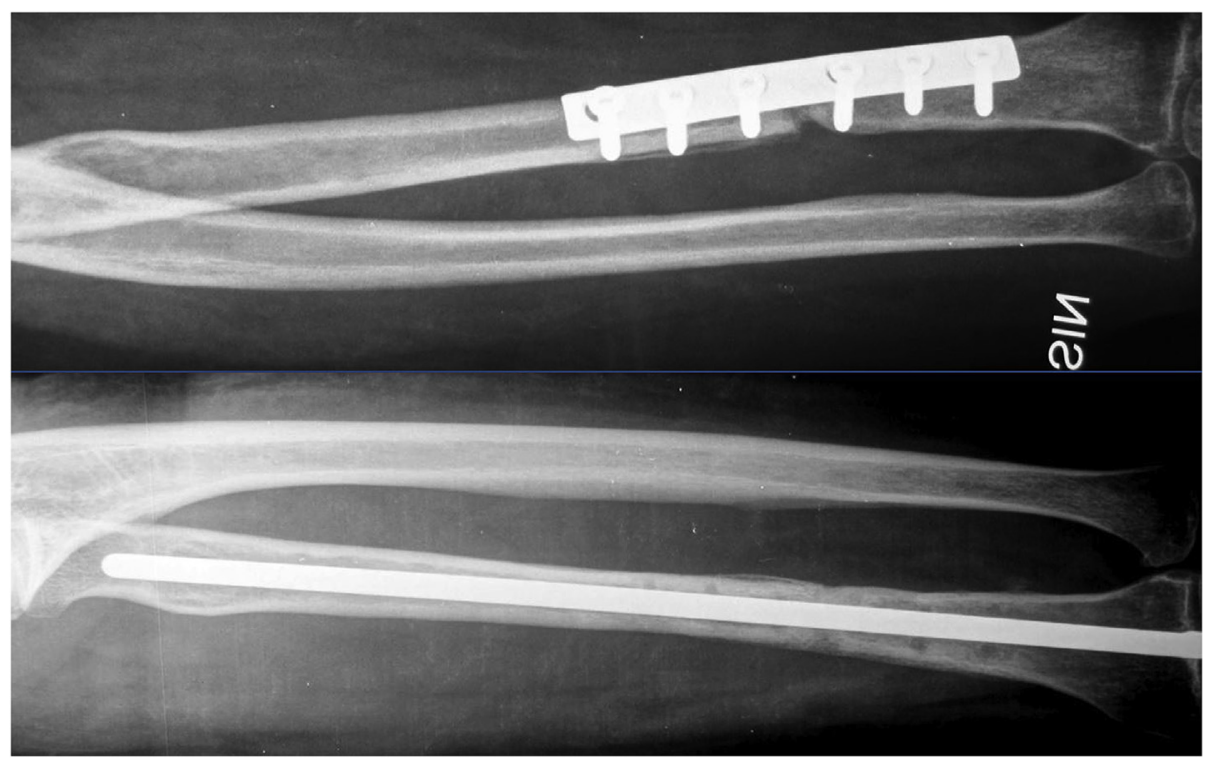

Fig. 1. Complete healing reached after plate removing and intramedullary nailing. 


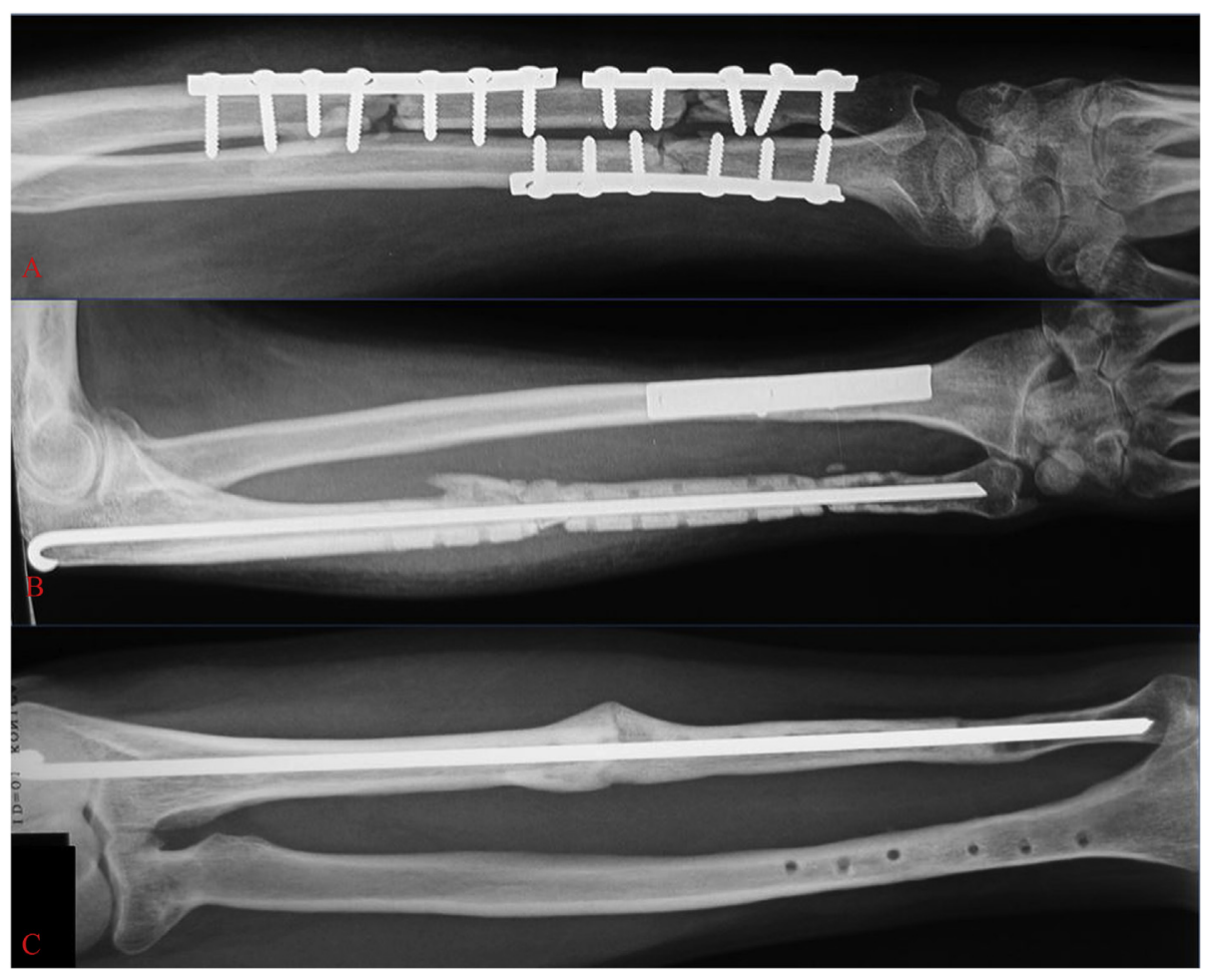

Fig. 2. Bifocal non-union of ulna (a). Radiographic assessment 3 months after surgery: bone graft was interposed at the proximal non-union site, and axial compression was performed using a nail (b). Radiographic assessment 6 months after surgery showing a complete healing of both bones (c).

ROM, an unsatisfactory result consists in healed fracture and $>30^{\circ}$ reduction of wrist and elbow ROM and $>50 \%$ reduction of forearm pronation-supination ROM. Failure consists in unhealed fracture (nonunion) and/or bony infection.

Disability-related quality of life was measured with the national validated version of disabilities of the arm, shoulder and hand (DASH) questionnaire, together with the optional module for assessment of working capacity (Work-DASH). ${ }^{22}$ This is a self-administered questionnaire which measures physical ability and symptoms of the upper extremity and explores the impact of functional impairment and pain on daily living tasks as well as social and recreational activities, work and sleep. The scoring system of the questionnaire is based on a metric scale ranging from 0 (minimum disability, best result) to 100 points (maximum disability, poorest result).

All the radiographic exams were independently evaluated by an orthopedic surgeon and a radiologist well-experienced in musculoskeletal imaging, and inter-observer reliability for assessment of bone healing was calculated. Clinical follow-up was scheduled for all the

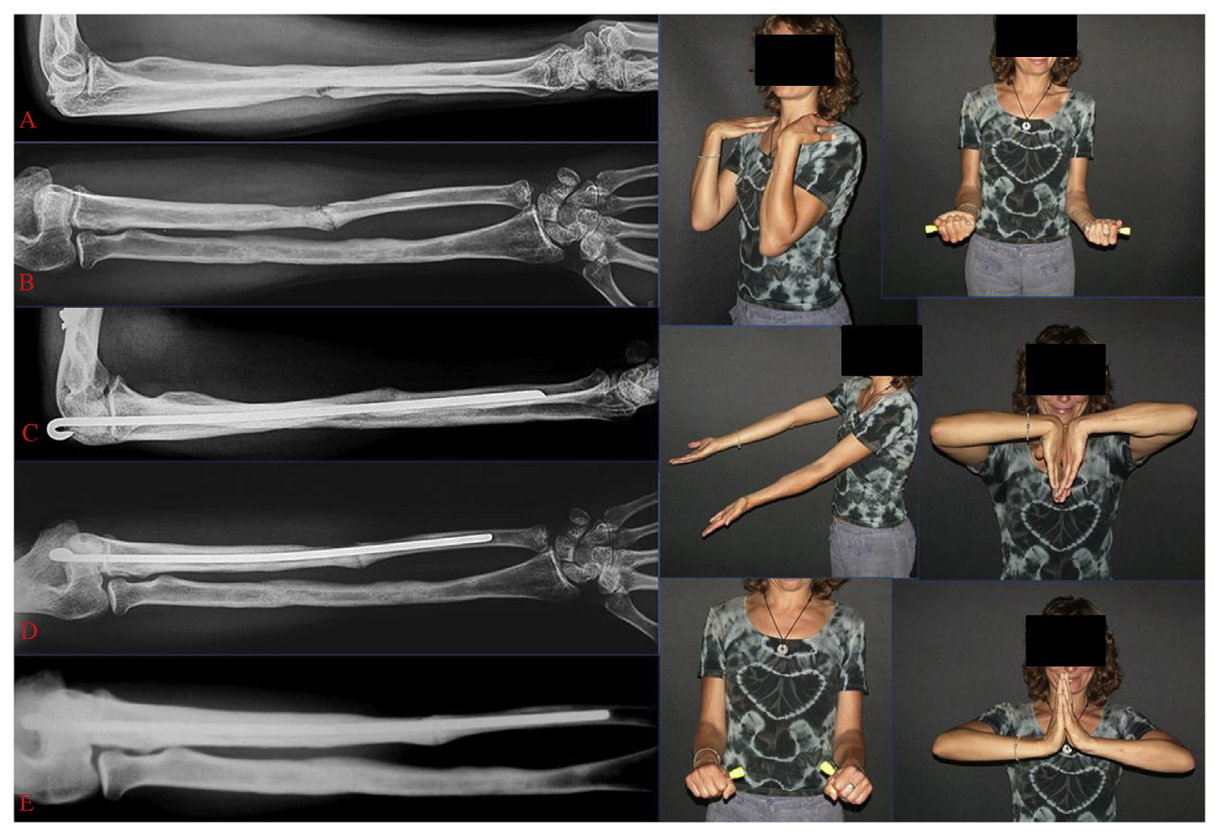

Fig. 3. A 35 years old patient with a longstanding ulna non-union (a, b). One month after intramedullary nailing (c, d): the nail fits perfectly inside the medullary canal. Eight months after surgery (e). On the right: final clinical results. 
patients at two years after surgery. Outcome measures were collected at follow-up visits by an independent investigator.

\subsection{Statistical analysis}

The study was designed as non-inferiority study. We compared our results with the average data reported in the literature for reference standard: open reduction and internal fixation (ORIF) using a compression plate fixation and autologous bone graft. ${ }^{3,6,19,20}$ Patients which showed treatment failure or postoperative complications and were referred to further treatments were evaluated according to the intention-to-treat-principles.

Statistical analysis was performed using the SPSS v.19.0 software (SPSS Inc.; Chicago, IL).

Inter-rater reliability for assessment of non-union healing was assessed by calculating Cohen's kappa coefficient on all the radiographic exams obtained during repeated evaluations.

Primary outcome results were statistically analyzed using the Chisquare test to compare our results with the data reported in the literature. $3,6,19,20$

For all tests, significance was set at $p<0.05$. Data were presented as mean \pm standard deviation (SD) for continuous variables and frequency and percentage for categorical variables.

\section{Results}

A total of 58 patients were consecutively evaluated and 49 (34 males and 15 females) were assessed as eligible. Seven patients were excluded because affected by other fractures on the same limb, 2 patients refused to enter the study. All the enrolled patients were reviewed at follow-up. Results are resumed in Table 1.

The average age of participants was $37.0(S D \pm 13.4)$ years. In 13 cases both bones (ulna and radius) were affected by non-union, in 18 cases only the radius, in 18 cases only the ulna. The dominant limb was affected in 25 cases. Bone grafting was used in 10 cases (atrophic nonunions). The average time passed from fracture was $14.9(\mathrm{SD} \pm 16.4)$ months. The mean follow-up was 31.8 months (SD \pm 23.0). Healing was reached in 46 cases (93.9\%), and this results are not significantly different from the average data reported in the literature $(\mathrm{p}>0.05)$. The average healing time was $6.3(\mathrm{SD} \pm 2.5)$ months.

Failure of non-union healing was observed only in 3 cases (6.1\%). In 2 cases $(4.1 \%)$, intramedullary nailing failed in a pseudarthrosis previously complicated by osteomyelitis, because a recurrence of infection. In one case $(2 \%)$ a recurrence of Complex Regional Pain Syndrome (CRPS) was observed: the person in question previously underwent firstly to an external fixation, later ORIF with plate and screw, but unsuccessfully; finally he underwent ORIF with plate and screw and iliac crest graft, which failed again.

At the last follow up ROM was calculated: the average pronationsupination ROM was 143.7 (SD \pm 24.7 ), the average flexion-extension ROM of the wrist was 150.0 (SD \pm 25.2 ), the average flexion-extension ROM of the elbow was 132.7 (SD \pm 13.0).

Anderson score was calculated: excellent result was reached in 38 cases $(77.6 \%)$, satisfactory in 7 cases $(14.3 \%)$, unsatisfactory in 1 case (2\%), failure in 3 cases $(6.1 \%)$.

At the most recent follow-up, the average score of DASH was 13.9 (SD \pm 17.2 ), and the average score of Work-DASH was 7.6 $(\mathrm{SD} \pm 10.2)$.

\subsection{Complications}

We never observed recurrence of fracture either before or after nail removal. Delayed healing requiring association with extracorporeal shock wave therapy (ESWT) was reported in five cases (10.2\%). In four cases $(8.2 \%)$, non-union of the radius healed with a shortening, thus producing ulno-carpal conflict which required subsequent resection of ulnar head (Darrach procedure) to restore adequate protonation and supination.

In one case rupture of the extensor pollicis longus occurred because of conflict with the curved extremity of the nail at critical point of Lister's tubercle. In this case, muscle-tendinous transfer of extensor indicis proprius (EIP) pro EPL was necessary.

Hardware migration was observed in one case, which required revision surgery by using a larger nail. No deep or superficial infections were observed, apart from two cases, in which further surgery was necessary.

\section{Discussion}

There is a lack of consensus about the definition of fracture-healing in the current orthopedic literature. Without valid and reliable clinical or radiographic measures of union, the interpretation of fracture care studies remains difficult. Variables used in the assessment of fracture healing are: (a) callus size; (b) cortical continuity; (c) progressive loss of fracture line; and (e) pain to palpation at the fracture site but use of only radiographic criteria in fracture healing studies should be abandoned in favor of a combination of radiographic and clinical measures. ${ }^{23,24}$

The treatment of forearm non-unions continues to represent a therapeutic challenge, and reported outcomes are moderate at best, with severe complications associated. ${ }^{15,19,25}$

In treatment of forearm non-union, the gold standard is an anatomic reconstruction and a complete recovery of function in both flexionextension and pronation-supination. ${ }^{5}$

Intramedullary osteosynthesis is very often considered a second choice, because, for many authors, nails do not guarantee rotational stability and osteosynthesis with plate and screws has become the treatment of choice for fracture-non-unions of the forearm. ${ }^{3,5,20}$ The Authors has reviewed literature and performed a retrospective evaluation of 49 forearm non-union with a minimal follow up of 1 years, demonstrating that intramedullary nailing of forearm non-union with Rush's nails has uses of fluoroscopy as only one disadvantage and has different advantages: is a mini-invasive technique with very small incisions and missed exposure of fracture, the extremely contained cost in comparison to the others technique, and, in experienced hands, the constant consolidation of non-union.

The analysis of case studies on this original surgery allows some final comments. Treatment of mono- or biosseous forearm non-union is particularly difficult as demonstrated by the case in existing literature that reports a relevant incidence of failures. ${ }^{3,6-8,10-18}$ The surgical procedure most commonly used in literature is represented by bone grafting and osteosynthesis with plate and screws. ${ }^{6,7,9-18}$ Plating, as we observed and as evidenced in literature, with a not infrequent intraoperative bad compression and its persistence over time, produces risk of not consolidation and break of the plate, or can make cortical bone like cancellous bone, and this adulterated quality of bone produce risk of a new fracture after removing plate and screws. ${ }^{25,26}$ Also exposition of fracture-non-union's site produce a risk of infection more elevated than intramedullary nailing and then osteomyelitis and early need of removing plate and screws without consolidation.

The intramedullary nailing by Rush's nail designed and used by Authors is the overcoming of these problems. In our experience, it was shown free from disadvantages and not inferior to he average data reported in the literature (Table 2). , $6,19,20^{-1}$

To perform intramedullary nailing, we used Rush nails with a diameter never inferior to $3.5 \mathrm{~mm}$. If possible the diameter and the length of the nail should be established before the operation. However, in ours methodic the apparently disproportionate diameter of the nail, never inferior to $3.5 \mathrm{~mm}$, conferred a continuity cortical-cortical and it was a fundamental element in confer rotational stability to the synthesis. The relative flexibility of the nail of Rush allowed such mean of synthesis to suit for the physiological bending of the forearm's bones. 


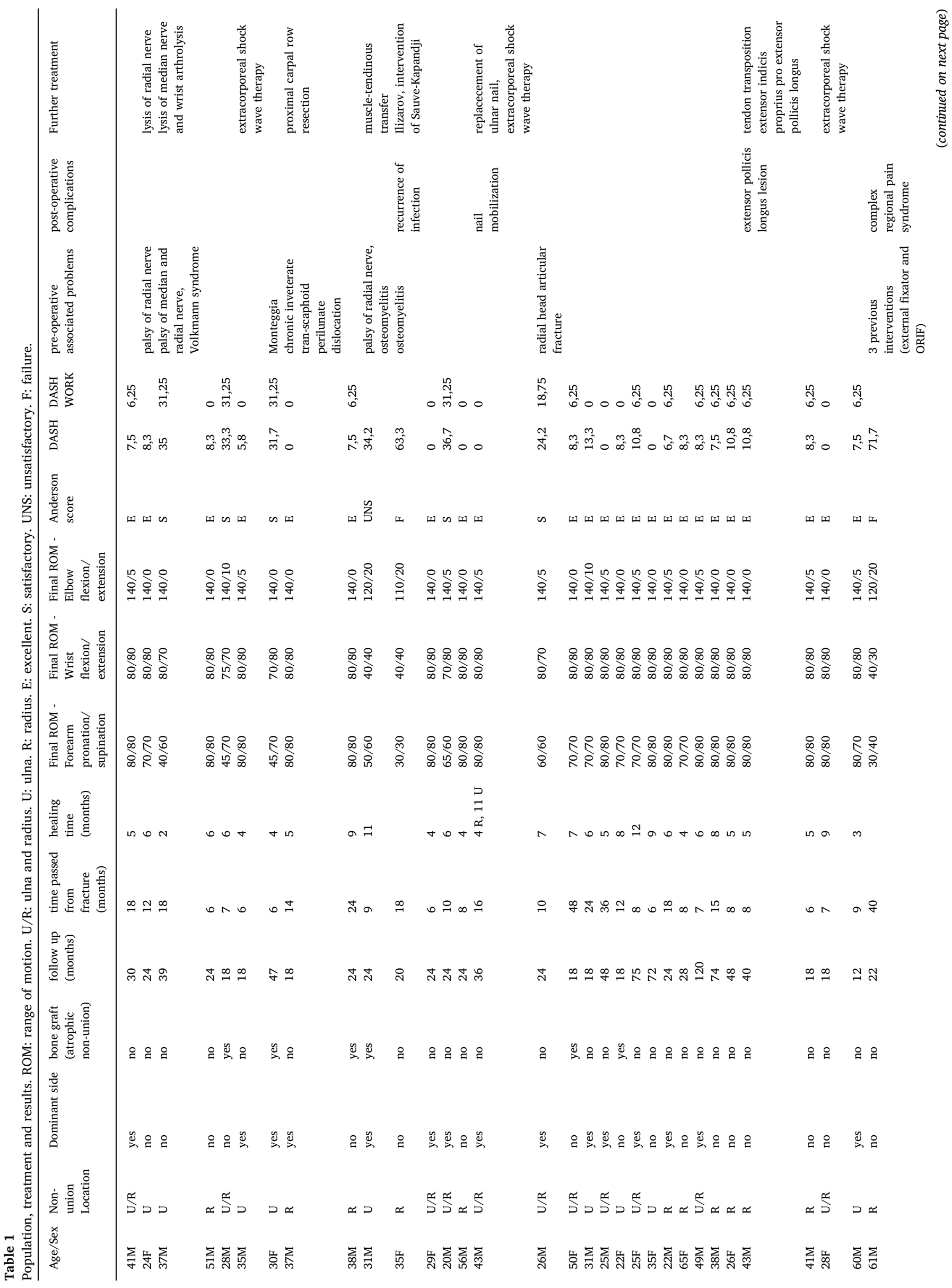




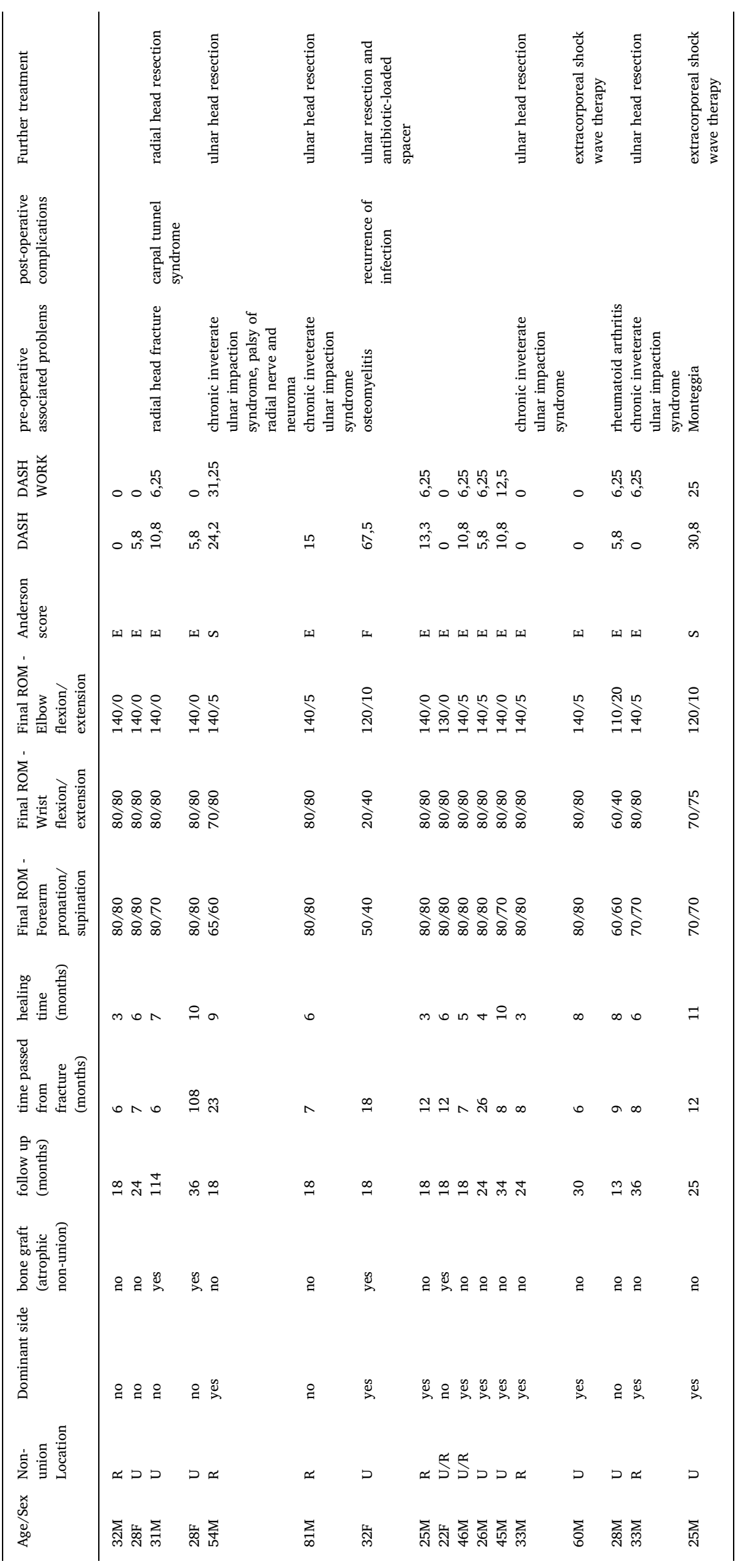


Table 2

Comparative results of forearm non-union treatment in other case series.

\begin{tabular}{|c|c|c|c|c|}
\hline Authors & Thechnique & Number of cases & Healing (\%) & Failure (\%) \\
\hline${ }^{6}$ Ring 2004 & ORIF (plate and screw) and bone grafting & 35 patients ( 16 radius, 11 ulna, 8 both) & 100 & 0 \\
\hline${ }^{19}$ Dos Reis 2009 & ORIF (plate and screw) and bone grafting & 31 patients ( 11 radius, 9 ulna, 11 both) & 96,8 & 3,2 \\
\hline${ }^{3}$ Kloen 2010 & ORIF (plate and screw) and eventually bone grafting & 47 patients with 51 non-unions & 100 & 0 \\
\hline${ }^{20}$ Boussakri 2016 & ORIF (plate and screw) and bone grafting & 21 patients with 26 non-unions & 95,1 & 4,9 \\
\hline Our study & intramedullary nailing and eventually bone grafting & 49 patients ( 18 radius, 18 ulna, 13 both) & 94,9 & 6,1 \\
\hline
\end{tabular}

Regarding to the length, it was necessary to synthesize the nonunion with a maximum length possible nail to obtain an optimal stability of the stumps.

There is an usual theoretical challenge about intramedullary nailing and Rush's nails that, according to many authors, presents the disadvantage of poor stability control, ${ }^{20}$ but has not found in our experience. We believe that this discrepancy arises from a pure mechanical reasoning, and our practice shows that, helped by cast for 30 days, this osteosynthesis obtain an excellent stability.

This explanation probably lies in some elements:

(a) use of high-diameter nails that involve a primary cortical to cortical taking;

(b) a synthesis of suitable length, such as Rush's nail, do not force to take a non-rectilinear geometry and support the physiological scoliosis of the two bones of the forearm, also at the moments of rotation;

(c) the minimal stabilizing effect in the rotation performed by the "curl" of Rush's nail, firmly fixed into the olecranon for ulna and into distal radial metaepiphysis at tubercle of Lister for radius.

The advantages of a closed nailing are a more rapid healing, greater stability for respect of soft tissues, better blood circulation to periosteum level, a less blood loss.

Bone grafting is the gold standard and it is need when there is a significant loss of bone tissue (atrophy) and consequently an important shortening. Furthermore bone graft can be shaped as tubular, tricortical or in the proper way to fill the gap. . $^{3,6-8,10,11,14-18,27}$

However, in other conditions we can use nothing or growth factors. The use of growth factors is not free from complication.PRP, BMP-2 and BMP-7 have been approved for clinical application in non-union bone fractures. An important prospective randomized clinical study demonstrated efficacy of rhBPM-7 an PRP in healing of long bone non-unions. ${ }^{28}$ However, has been described some difficulties of using the dosedependent properties of BMP like a recent massive inflammatory reaction after use of recombinant human BMP-2 for repair of a symptomatic ulnar non-union in a child. ${ }^{29}$ Also, a systematic review described conflicting results in use of PRP probably due to preparation. ${ }^{30}$

Another great problem in the use of the growth factors is the elevated costs of a therapy that do not have a documented effectiveness. In a perspective of cost containment, a pressing problem to which no department director can escape, it is unthinkable that this type of therapy can be used on a large scale.

\section{Conclusions}

The treatment of forearm fracture-non-unions continues to represent a therapeutic challenge. Although osteosynthesis with plate and screws has become the treatment of choice for fracture-non-union of the forearm intramedullary osteosynthesis is a low-cost alternative treatment that ever have been considered, but especially when is possible to perform an osteosynthesis without exposure of non-union's site.

\section{Source of funding}

The authors report no source of funding.

\section{Declaration of competing interest}

The authors report no conflicts of interest.

\section{References}

1. Wright RR, Schmeling GJ, Schwab JP. The necessity of acute bone grafting in diaphyseal forearm fractures: a retrospective review. J Orthop Trauma. 1997;11(4):288-294.

2. Wei SY, Born CT, Abene A, Ong A, Hayda R, DeLong WGJ. Diaphyseal forearm fractures treated with and without bone graft. J Trauma. 1999;46(6):1045-1048. https://doi.org/10.1097/00005373-199906000-00011.

3. Kloen P, Wiggers JK, Buijze GA. Treatment of diaphyseal non-unions of the ulna and radius. Arch Orthop Trauma Surg. 2010;130(12):1439-1445. https://doi.org/10. 1007/s00402-010-1071-x.

4. Street DM. Intramedullary forearm nailing. Clin Orthop Relat Res. 1986(212):219-230

5. Anderson LD, Sisk D, Tooms RE, Park 3rd WI. Compression-plate fixation in acute diaphyseal fractures of the radius and ulna. J Bone Joint Surg Am. 1975;57(3):287-297.

6. Ring D, Allende C, Jafarnia K, Allende BT, Jupiter JB. Ununited diaphyseal forearm fractures with segmental defects: plate fixation and autogenous cancellous bonegrafting. J Bone Joint Surg Am. 2004;86(11):2440-2445.

7. Moroni A, Rollo G, Guzzardella M, Zinghi G. Surgical treatment of isolated forearm non-union with segmental bone loss. Injury. 1997;28(8):497-504. https://doi.org/ 10.1016/s0020-1383(97)00044-2.

8. Spira E. Bridging of bone defects in the forearm with iliac graft combined with intramedullary nailing. J Bone Joint Surg Br. 1954;36-B(4):642-646.

9. Niccol E. The treatment of gaps in long bones by cancellous insert grafts. $J$ Bone Joint Surg Br. 1956;38-B(1):70-82.

10. Scaglietti O, Stringa G, Mizzau M. Bone grafting in nonunion of the forearm. Clin Orthop Relat Res. 1965;43:65-76. https://doi.org/10.1097/00003086-196500430 00007.

11. Moroni A, Caja VL, Sabato C, Rollo G, Zinghi G. Composite bone grafting and plate fixation for the treatment of nonunions of the forearm with segmental bone loss: a report of eight cases. J Orthop Trauma. 1995;9(5):419-426.

12. Hong G, Cong-Feng L, Hui-Peng S, Cun-Yi F, Bing-Fang Z. Treatment of diaphyseal forearm nonunions with interlocking intramedullary nails. Clin Orthop Relat Res. 2006;450:186-192. https://doi.org/10.1097/01.blo.0000214444.87645.75.

13. Christensen NO. Kuntscher intramedullary reaming and nail fixation for nonunion of the forearm. Clin Orthop Relat Res. 1976;116:215-221.

14. Gupta DK, Kumar G. Gap nonunion of forearm bones treated by modified Nicoll's technique. Indian J Orthop. 2010;44(1):84-88. https://doi.org/10.4103/0019-5413. 58611.

15. Faldini C, Pagkrati S, Nanni M, Menachem S, Giannini S. Aseptic forearm nonunions treated by plate and opposite fibular autograft strut. Clin Orthop Relat Res. 2009;467(8):2125-2134. https://doi.org/10.1007/s11999-009-0827-5.

16. Faldini C, Miscione MT, Acri F, Chehrassan M, Bonomo M, Giannini S. Use of homologous bone graft in the treatment of aseptic forearm nonunion. Musculoskelet Surg. 2011;95(1):31-35. https://doi.org/10.1007/s12306-011-0117-8.

17. Miller RC, Phalen GS. The repair of defects of the radius with fibular bone grafts. $J$ Bone Joint Surg Am. 1947;29(3):629-636.

18. Jupiter JB, Gerhard HJ, Guerrero J, Nunley JA, Levin LS. Treatment of segmental defects of the radius with use of the vascularized osteoseptocutaneous fibular autogenous graft. J Bone Joint Surg Am. 1997;79(4):542-550. https://doi.org/10.2106/ 00004623-199704000-00009.

19. dos Reis FB, Faloppa F, Fernandes HJA, Albertoni WM, Stahel PF. Outcome of diaphyseal forearm fracture-nonunions treated by autologous bone grafting and compression plating. Ann Surg Innovat Res. 2009;3:5. https://doi.org/10.1186/1750 1164-3-5.

20. Boussakri H, Elibrahimi A, Bachiri M, Elidrissi M, Shimi M, Elmrini A. Nonunion of fractures of the ulna and radius diaphyses: clinical and radiological results of surgical treatment. Malaysian Orthop J. 2016;10(2):27-34. https://doi.org/10.5704/MOJ. 1607.006 .

21. Bhandari M, Guyatt GH, Swiontkowski MF, Tornetta 3rd P, Sprague S, Schemitsch $\mathrm{EH}$. A lack of consensus in the assessment of fracture healing among orthopaedic surgeons. J Orthop Trauma. 2002;16(8):562-566

22. Padua R, Padua L, Ceccarelli E, et al. Italian version of the Disability of the Arm, Shoulder and Hand (DASH) questionnaire. Cross-cultural adaptation and validation. $J$ Hand Surg Br. 2003;28(2):179-186.

23. Dijkman BG, Sprague S, Schemitsch EH, Bhandari M. When is a fracture healed? 
Radiographic and clinical criteria revisited. J Orthop Trauma. 2010;24(Suppl 1):S76-S80. https://doi.org/10.1097/BOT.0b013e3181ca3f97.

24. Corrales LA, Morshed S, Bhandari M, Miclau 3rd T. Variability in the assessment of fracture-healing in orthopaedic trauma studies. J Bone Joint Surg Am. 2008;90(9):1862-1868. https://doi.org/10.2106/JBJS.G.01580.

25. Stern PJ, Drury WJ. Complications of plate fixation of forearm fractures. Clin Orthop Relat Res. 1983;175:25-29.

26. Mih AD, Cooney WP, Idler RS, Lewallen DG. Long-term follow-up of forearm bone diaphyseal plating. Clin Orthop Relat Res. 1994;299:256-258.

27. De Vitis R, Passiatore M, Perna A, Tulli A, Pagliei A, Taccardo G. Modified MattiRusse technique using a "butterfly bone graft" for treatment of scaphoid non-union. $J$
Orthop. 2019. https://doi.org/10.1016/j.jor.2019.11.030.

28. Calori GM, Tagliabue L, Gala L, D'Imporzano M, Peretti G, Albisetti W. Application of rhBMP-7 and platelet-rich plasma in the treatment of long bone non-unions: a prospective randomised clinical study on 120 patients. Injury. 2008;39(12):1391-1402. https://doi.org/10.1016/j.injury.2008.08.011.

29. Ritting AW, Weber EW, Lee MC. Exaggerated inflammatory response and bony resorption from BMP-2 use in a pediatric forearm nonunion. $J$ Hand Surg Am. 2012;37(2):316-321. https://doi.org/10.1016/j.jhsa.2011.10.007.

30. Griffin XL, Smith CM, Costa ML. The clinical use of platelet-rich plasma in the promotion of bone healing: a systematic review. Injury. 2009;40(2):158-162. https:// doi.org/10.1016/j.injury.2008.06.025 